\title{
A Physics Modeling Study of COVID-19 Transport in Air
}

\author{
Luis A. Anchordoqui a ${ }^{*}{ }^{*}$, James B. Dent ${ }^{c}$, Thomas J. Weiler ${ }^{\text {d }}$ \\ ${ }^{a}$ Department of Physics and Astronomy, Lehman College, City University of New York, NY 10468, United States. \\ ${ }^{b}$ Department of Physics, Graduate Center, City University of New York, NY 10016, United States. \\ ${ }^{c}$ Department of Physics, Sam Houston State University, Huntsville, TX 77341, United States. \\ ${ }^{d}$ Department of Physics and Astronomy, Vanderbilt University, Nashville, TN 37235, United States.
}

Received 16 July 2020; Accepted 02 October 2020

\begin{abstract}
Objectives: Health threat from COVID-19 airborne infection has become a public emergency of international concern. During the ongoing coronavirus pandemic, people have been advised by the Centers for Disease Control and Prevention to maintain social distancing of at least $2 \mathrm{~m}$ to limit the risk of exposure to the coronavirus. Experimental data, however, show that infected aerosols and droplets trapped inside a turbulent puff cloud can travel 7 to $8 \mathrm{~m}$. We carry out a physics modeling study for COVID-19 transport in air. Methodology: We propose a nuclear physics analogy-based modeling of the complex gas cloud and its payload of pathogen-virions. We estimate the puff effective stopping range adapting the high-energy physics model that describes the slow down of $\alpha$-particles (in matter) via interactions with the electron cloud. Analysis Findings: We show that the cloud stopping range is proportional to the diameter of the puff times its density. We use our puff model to determine the average density of the buoyant fluid in the turbulent cloud. A fit to the experimental data yields $1.8<\rho_{P} / \rho_{\text {air }}<4.0$, where $\rho_{P}$ and $\rho_{\text {air }}$ are the average density of the puff and the air. We demonstrate that temperature variation could cause an $O(\leqslant \pm 8 \%)$ effect in the puff stopping range for extreme ambient cold or warmth. We also demonstrate that aerosols and droplets can remain suspended for hours in the air. Therefore, once the puff slows down sufficiently, and its coherence is lost, the eventual spreading of the infected aerosols becomes dependent on the ambient air currents and turbulence.
\end{abstract}

Keywords: COVID-19; SARS-CoV-2; Transport; Airborne Infection; Aerosol.

\section{Introduction}

The current outbreak of the respiratory disease identified as COVID-19 is caused by the severe acute respiratory syndrome coronavirus 2, shortened to SARS-CoV-2 [1-4]. The outbreak was first reported in December 2019, and has become a worldwide pandemic with over 10 million cases as of 1 July 2020. SARS-CoV-2 have been confirmed worldwide and so the outbreak has been declared a global pandemic by the World Health Organization. The pandemic has spread around the globe to almost every region, with only a handful of the World Health Organization's member states not yet reporting cases. Most of these states are small island nations in the Pacific Ocean, including Vanuatu, Tuvalu, Samoa, and Palau.

The coronavirus can spread from person-to-person in an efficient and sustained way by coughing and sneezing. The virus can spread from seemingly healthy carriers or people who had not yet developed symptoms [5]. To understand and prevent the spread of the virus, it is important to estimate the probability of airborne transmission as aerosolization with particles potentially containing the virus. Before proceeding, we pause to note that herein we

* Corresponding author: luis.anchordoqui@lehman.cuny.edu

$>$ This is an open access article under the CC-BY license (https://creativecommons.org/licenses/by/4.0/).

(C) Authors retain all copyrights. 
follow the convention of the World Health Organization and refer to particles which are $\gtrsim 5 \mu m$ diameter as droplets and those $\leqslant 5 \mu m$ as aerosols or droplet nuclei [6].

There are various experimental measurements suggesting that SARS-CoV-2 may have the potential to be transmitted through aerosols; see e.g. [7-11]. Indeed, laboratory-generated aerosols with SARS-CoV-2 were found to keep a replicable virus in cell culture throughout the 3 hours of aerosol testing [12]. Of course these laboratorygenerated aerosols may not be exactly analogous to human exhaled droplet nuclei, but they helped in establishing that the survival times of SARS-CoV-2 depend on its environment, including survival times of: up to 72 hours on plastics, up to 48 hours on stainless steel, up to 24 hours on cardboard, up to 4 hours on copper, and in air for 3 to 4 hours [12]. On first glimpse this finding is surprising, as one would expect that the properties of air that degrade the SARS-CoV-2 exterior should abate at roughly half that time if it were adhered to a surface (i.e. at least half the solid angle is mostly exposed to air). However, the laboratory-generated aerosols have shown that a precise description of SARS-CoV-2 main characteristics requires more complex systems in which the virus would be chemisorbed by some surfaces and repelled by the others. More concretely, the survival probability of the virus is associated with the surface energies of the various materials that can reduce the solid angle exposed to air molecule collisions. These proper- ties can lead to remarkable differences, for example that between copper and stainless steel. Despite the fact both are metals, copper causes destruction of the virus much more rapidly than does stainless steel.

The number of virions needed for infection is yet un- known. However, it is known that viral load differs considerably between SARS-CoV and SARS-CoV-2 [13]. A study of the variance of viral loads in patients of different ages found no significant difference between any pair of age categories including children [14].

Beyond a shadow of a doubt, a major question of this pandemic has been how far would be far enough to elude droplets and to diffuse droplet nuclei if a person nearby is coughing or sneezing. The rule of thumb for this pandemic has been a $2 \mathrm{~m}$ separation. Nevertheless, this has never been a magic number that guarantees complete protection. Indeed, experiment shows that: (i) respiratory particles emitted during a sneeze or cough are initially transported as a turbulent cloud that consists of hot and moist exhaled air and mucosalivary filaments; (ii) aerosols and small droplets trapped in the turbulent puff cloud could propagate 7 to $8 \mathrm{~m} \mathrm{[15-18].} \mathrm{Moreover,} \mathrm{once} \mathrm{the} \mathrm{cloud} \mathrm{slows} \mathrm{down}$ sufficiently, and its coherence is lost, the eventual spreading of the infected aerosols becomes dependent on the ambient air currents and turbulence [19]. In this paper we provide new guidance to address this question by introducing a physics model for SARS-CoV-2 transport in air.

To develop some sense for the orders of magnitude involved, we begin by reviewing the experimental data. A survey of 26 analyses reporting particle sizes generated from breathing, coughing, sneezing and talking indicates that healthy individuals generate particles with sizes in the range $0.01 \leqslant D_{V} / \mu m \leqslant 500$, whereas individuals with infections produce particles in the range $0.05 \leqslant D_{V} / \mu m \leqslant 500$, where $D_{V}$ is the diameter of a respiratory particle (droplet or droplet nucleus) containing the virus [20]. The majority of the particles containing the virus have outlet velocities in the range $10 \leqslant v_{V, 0} /(\mathrm{m} / \mathrm{s}) \leqslant 30[18,21,22]$. Up to $10^{4.6}$ particles are expelled at an initial velocity of $30 \mathrm{~m} / \mathrm{s}$ during a sneeze, and a cough can generate approximately $10^{3.5}$ particles with outlet velocities of $20 \mathrm{~m} / \mathrm{s}$ [23]. 97\% of coughed particles have sizes $0.5 \leq D_{V} / \mu m \lesssim 12$, and the primary size distribution is within the range $1 \leq D_{V} / \mu m \lesssim 2$ [24, 25]. The evaporation rate of the respiratory particles depends on the exposed surface area, $A \sim \pi D_{V}^{2}$, while the particle's volume scales as $V \sim \pi D_{V}^{3} / 6$. Therefore, the ratio of area to volume is $A / V \propto 1 / D_{V}$, and it is the smallest droplets that will live the longest.

The layout of the paper is as follows. In Sec. II we review the generalities of aerodynamic drag force and estimate the terminal speed of aerosols and droplets. In Sec. III we model the elastic scattering of the turbulent cloud with the air molecules and estimate the puff stop- ping range assuming standard ambient temperature and pressure conditions. After that, we use our puff model to determine the average density of the buoyant fluid in the turbulent cloud. The paper wraps up with some conclusions presented in Sec. IV.

\section{Terminal Speed}

When a particle propagates through the air, the surrounding air molecules have a tendency to resist its motion. This resisting force is known as the aerodynamic drag force. For a spherical particle, the aerodynamic drag force is given by:

$$
\mathbf{F}_{d}=3 \pi \eta_{\text {air }} D_{V} \mathbf{v}_{V} \frac{1}{\kappa}
$$

Where $\eta_{\text {air }} \simeq 1.8 \times 10^{-5} \mathrm{~kg} /(\mathrm{m} . \mathrm{s})$ is the dynamic viscosity of air and $\mathbf{v}_{\mathrm{v}}$ is the virus velocity vector. Equation 1 is the well-known Stokes' law, with the Cunningham slip correction factor $\kappa$; see Appendix I for details. Stokes' law assumes that the relative velocity of a carrier gas at a particle's surface is zero; this assumption does not hold for small particles. The slip correction factor should be applied to Stokes' law for particles smaller than $10 \mu \mathrm{m}$. 
$\mathcal{R}=\frac{D_{V} v_{V} \rho_{\text {air }}}{\eta_{\text {air }}}$

The particle Reynolds number is a dimensionless quantity which represents the ratio of inertial forces to viscous forces, where $\rho_{\text {air }} \simeq 1.2 \mathrm{~kg} / \mathrm{m}^{3}$ is the air density at a temperature of $20^{\circ} \mathrm{C}(293 \mathrm{~K})$. For $\mathcal{R}<1$, the inertial forces can be neglected. The drag calculated by Equation 1 has an error of about $12 \%$ at $\mathcal{R} \approx 1$. The error decreases with decreasing particle Reynolds number.

For the case at hand, $\mathcal{R}>1$. In the vertical direction, the upward component of the aerodynamic drag force $F_{d}$ is counterbalanced by the excess of the gravitational attraction over the air buoyancy force:

$F_{g}=\frac{1}{6} \pi D_{V}^{3}\left(\rho_{\mathrm{H}_{2} \mathrm{O}}-\rho_{\text {air }}\right) g$

Where $\rho_{\mathrm{H}_{2} \mathrm{O}} \simeq 997 \mathrm{~kg} / \mathrm{m}^{3}$ and $g \simeq 9.8 \mathrm{~m} / \mathrm{s}^{2}$ is the acceleration of gravity. Since $\rho_{\text {air }} \ll \rho_{\mathrm{H}_{2} \mathrm{O}}$ the air buoyancy force becomes negligible, and so $F_{g} \approx M_{V} g$, with $M_{V}$ the aerosol mass. When the upward aerodynamic drag force equals the gravitational attraction the droplet reaches mechanical equilibrium and starts falling with a terminal speed:

$v_{V, f \downarrow} \approx \frac{M_{V} g \kappa}{3 \pi \eta D_{V}}$

The terminal speed is $\propto D_{V}^{2}$ (due to the diameter dependence of the mass), and hence larger droplets would have larger terminal velocities thereby reaching the ground faster. The terminal speed for various particle sizes is given in Table 1 . The time $t_{f}$ it will take the virus to fall to the ground is simply given by the distance to the ground divided by $v_{V, f, \cdot}$. For an initial height, $h \sim 2 \mathrm{~m}$, we find that for $D_{V}=2 \mu \mathrm{m}$,

$t_{f}=\frac{h}{v_{V, f, \perp}} \sim 4 \mathrm{hr}$

The time scale as a function of the droplet size and height is shown in Figure 1.

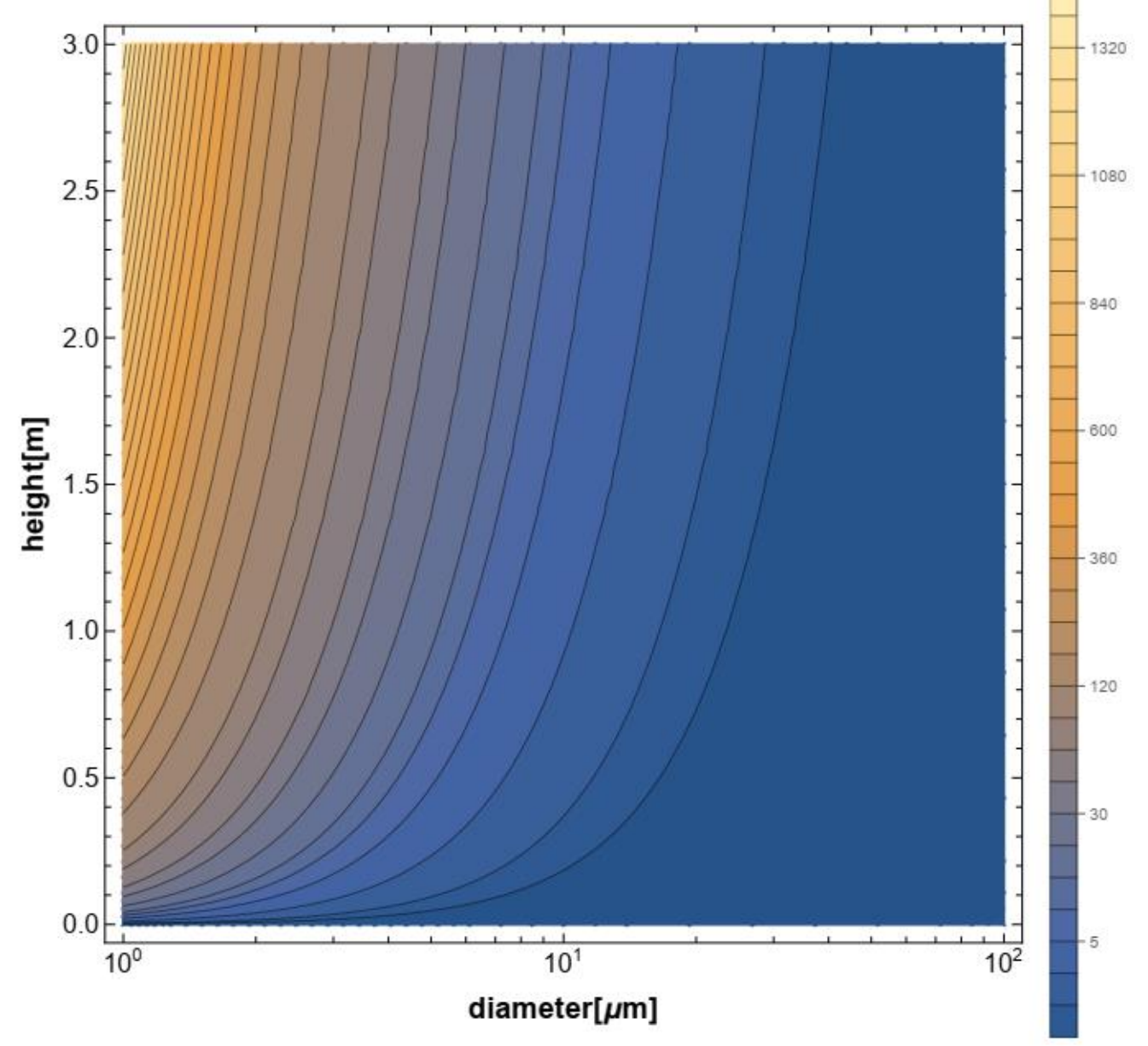

Figure 1. Contours of the time $t_{f}$ in minutes in the $h-D_{v}$ plane 
Table 1. Cunningham slip correction factor and terminal speed

\begin{tabular}{ccc}
\hline $\mathbf{D}_{\mathbf{V}}(\mu \mathbf{m})$ & $\kappa$ & $\boldsymbol{v}_{\mathbf{V}, \mathbf{f}, \perp}(\mathbf{m} / \mathbf{s})$ \\
\hline 0.001 & 215.3 & $6.51 \times 10^{-9}$ \\
0.010 & 22.05 & $6.67 \times 10^{-8}$ \\
0.100 & 2.851 & $8.62 \times 10^{-7}$ \\
0.500 & 1.327 & $1.00 \times 10^{-5}$ \\
1.000 & 1.163 & $3.52 \times 10^{-5}$ \\
1.500 & 1.109 & $7.54 \times 10^{-5}$ \\
2.000 & 1.081 & $1.31 \times 10^{-4}$ \\
3.000 & 1.054 & $2.87 \times 10^{-4}$ \\
5.000 & 1.033 & $7.81 \times 10^{-4}$ \\
7.000 & 1.023 & $1.52 \times 10^{-3}$ \\
10.000 & 1.016 & $3.07 \times 10^{-3}$ \\
\hline
\end{tabular}

The aerodynamic drag force holds for rigid spherical particles moving at constant velocity relative to the gas flow. To determine the stopping range, in the next section we model the elastic scattering of the turbulent puff cloud with the air molecules.

\section{Stopping Range}

Respiratory particles of saliva and mucus are expelled together with a warm and humid air, which generates a convective current. The aerosols and droplets are initially transported as part of a coherent gas puff of buoyant fluid. The ejected puff of air remains coherent in a volume that varies from 0.00025 to $0.0025 \mathrm{~m}^{3}$ [26]. This corresponds to a puff size $0.78 \leqslant D_{P} / m \leqslant 1.68$, where following [26] we have taken an entrainment coefficient of $\alpha=0.1$ [27]. The puff is ejected with $1 \leqslant v_{\mathrm{V}, 0, \|} /(\mathrm{m} / \mathrm{s}) \leqslant 10$ [26]. The turbulent puff cloud consists of an admixture of moist exhaled air and mucosalivary filaments. Next, in line with our stated plan, we use the experimental data to calculate the range of the average density of the buoyant fluid in the turbulent cloud.

The mass ratio of the average air molecule compared to the aerosol, $m_{\text {air }} / M_{V}$, is roughly $10^{-12}$ (since the size of the aerosol and the mass for its chief constituent, $\mathrm{H}_{2} \mathrm{O}$, compared to the air molecule are $10^{4}$ and $10^{3}$ ), though there is an obvious variation with aerosol size at constant density. If we consider instead the mass inside the puff $M_{P}$ the ratio $R \equiv m_{\text {air }} / M_{P}$ is even smaller. Due to the enormous mass ratio, the virions inside the puff will not undergo large angular deflections, so we will treat the virions as having the same direction for its initial and final velocities (since we are looking at a stopping distance, this is a reasonable assumption). Starting with the non-relativistic one-dimensional equation for the virus velocity $\beta$ we have in the lowest nontrivial order (in $\mathrm{R} \ll 1$ ) and any frame:

$\left(\begin{array}{c}\beta_{1} \\ v_{\text {air, } f}\end{array}\right)=\mathbf{M}\left(\begin{array}{c}\beta_{0} \\ v_{\text {air, }, 0}\end{array}\right)$

Where the matrix $\boldsymbol{M}$ is derived by imposing conservation of energy and momentum, and is given by:

$\mathbf{M}=\left(\begin{array}{cc}1-2 \mathrm{R} & 2 \mathrm{R} \\ 2 & -1\end{array}\right)$

With $\beta_{0}=v_{V, 0, \|}$, and $v_{a i r, 0}$ and $v_{\text {air, } f}$ the initial and final velocities of the air molecule, respectively. As the velocity $\beta$ falls with each interaction, the velocity loss remains constant; the target particle is a new air molecule at each interaction.

Though individual air molecules are traveling at an average speed of a few hundred meters per second, throughout we assume the medium to be stationary. In analogy with the description of the slowing down of alpha particles in matter (which assumes the electronic cloud is at rest), we can describe the scattering of the puff in the frame in which the air molecule is at rest, i.e., $v_{a i r, 0}=0$ (in essence, adopting a stationary medium on average). The stopping power is given by the velocity- loss equation: 
$d \beta / d x=\Delta \beta / \lambda_{\mathrm{mfp}}^{V}=2 R \beta / \lambda_{\mathrm{mfp}}^{V}$

With solution $\left.\ln \beta=\left(2 R / \lambda_{\mathrm{mfp}}^{V}\right)\right\rfloor d x$. Finally, we have for the stopping distance:

$L=\lambda_{\text {mp }}^{V} \frac{1}{2 R} \ln \left(\frac{\beta_{0}}{\beta_{f}}\right)$

With $\beta_{f}=v_{V, f, \|}$, Note that $L / \lambda_{m f p}^{V}$ is not only the number of mean free paths traversed by the fiducial virus, but is also the number of interactions of the virus with air molecules; of course, there is a one-to-one correlation between the number of mean free paths travelled and interactions.

Since $\beta$ is homogeneous and the mass ratio $R$ is a constant for a given puff size $D_{P}$, we have the above simple equation. The mass ratio $R$ is very small, and $(2 R)^{-1}$ is correspondingly very large. There are a tremendous number of mean free paths/interactions involved as the virions bowling ball rolls over the air molecule.

Finally, we must calculate $\lambda_{m f p}^{V}=1 /\left(\eta_{\text {air }} \sigma\right)$. The air molecules act collectively as a fluid, so the volume $\mathrm{V}$ over the air density is given by the ideal gas law as $k_{B} T / P$, where $P$ is the pressure, $T$ the temperature, and $k_{B}$ is the Boltzmann constant. We assume a contact interaction equal to the cross-sectional hard-sphere size of the puff, i.e. $\sigma=\pi\left(D_{P} / 2\right)^{2}$. Substituting into Equation 9 we obtain the final result for the stopping distance.

$L=\frac{k_{\mathrm{B}} T}{P} \frac{1}{\pi\left(D_{P} / 2\right)^{2}} \frac{1}{2 R} \ln \left(\frac{\beta_{0}}{\beta_{f}}\right)$

We take the sneeze or cough which causes the droplets expulsion to be at a standard ambient air pressure of $P=101 \mathrm{kPa}$ and a temperature of $T \sim 293 \mathrm{~K}$. It is important to stress that temperature variation could cause an $O($ $\leqslant \pm 8 \%$ ) effect in $L$ for extreme ambient cold or warmth. We now proceed to fit the experimental data. For $L \sim 8 m$ and taking $v_{V, f, \|} \sim 3 \mathrm{~mm} / \mathrm{s}$ [16], we obtain $1.8<\rho_{P} / \rho_{\text {air }}<4.0$ for $0.78 \leq D_{P} / m \leq 1.68$, where $\rho_{P}$ is the average density of the fluid in the puff.

A point worth noting at this juncture is that our model provides an effective description of the turbulent puff cloud. Note that independently of their size and their initial velocity all respiratory particles in the cloud experience both gravitational settling and evaporation. Aerosols and droplets of all sizes are subject to continuous settling, but those with settling speed smaller than the fluctuating velocity of the surrounding puff would remain trapped longer within the puff. Actually, because of evaporation the water content of the respiratory particles is monotonically decreasing. At the point of almost complete evaporation the settling velocity of the aerosols is sufficiently small that they can remain trapped in the puff and get advected by ambient air currents and dispersed by ambient turbulence. The size of the puff then continuously grows in time [26]. Our result can equivalently be interpreted in terms of the effective coherence length of the turbulent cloud assuming $\rho_{P} \sim \rho_{\text {air }}$. The effective size of the puff and its effective density are entangled in Equation 10. Numerical simulations show that during propagation the puff edge grows $\propto t^{1 / 4}$ [16]. After a100 s the puff would grow by a factor of 3 [26], in agreement with our analytical estimates. In closing, we note that if we ignore the motion of the air puff carrying the aerosols, as in the analysis of Wells (1934) [28], it is straightforward to see substituting $R$ by $m_{\text {air }} / M_{V} \sim 10^{-12}$ into Equation 10 that the individual aerosols would not travel more than a few $\mathrm{cm}$ away from the exhaler, even under conditions of fast ejections, such as in a sneeze. This emphasizes the relevance of incorporating the complete multiphase flow physics in the modeling of respiratory emissions when ascertaining the risk of SARS-CoV-2 air- borne infection. 


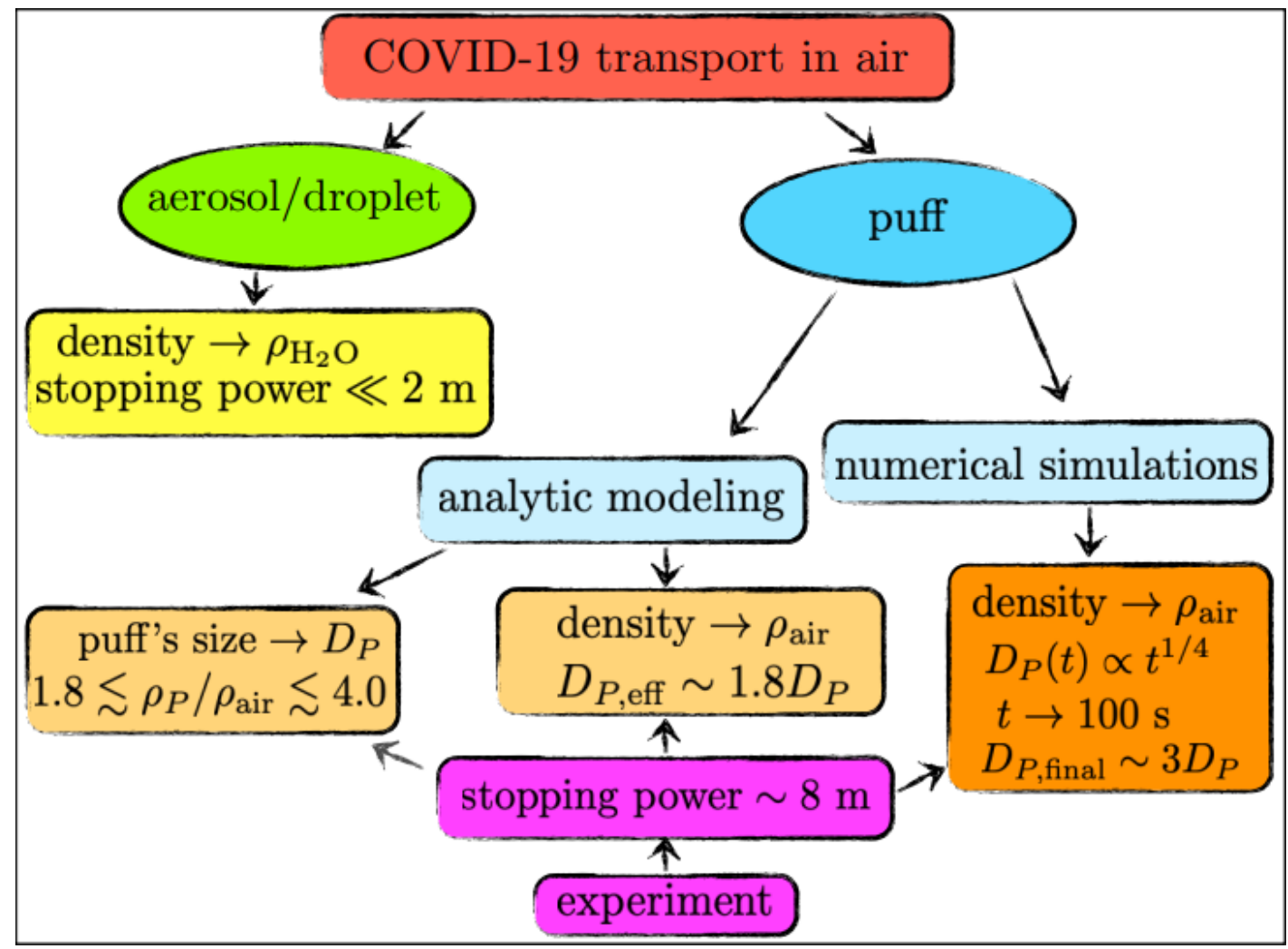

Figure 2. A flow chart showing the research methodology. The study of COVID-19 transport in air has been carried out using a modeling of the turbulent cloud. For a coherent length of $D_{P}$ the average density becomes $1.8<\rho_{P} / \rho_{\text {air }}<4.0$. For $\rho_{P} \sim \rho_{\text {air }}$ the effective size of the puff is $D_{P}$, eff $\sim 1.8 D_{P}$. The latter is consistent with simulation studies which show that the coherent length evolves in time proportional to $t^{1 / 4}$, and so after $100 \mathrm{~s}$ the final size of the coherent turbulent cloud is $D_{P, \text { final }} \sim 3 D_{P}$. Viral transmission pathways have profound implications for public safety. Our study forewarns a health threat of COVID-19 airborne infection in indoor spaces. We argue in favor of implementing additional precautions to the recommended $2 \mathrm{~m}$ social distancing, e.g. wearing a face mask when we are out in public.

\section{Conclusion}

We have carried out a physics modeling study for SARS-CoV-2 transport in air. We have developed a nuclear physics analogy-based modeling of the complex gas cloud and its payload of pathogen-virions. Using our puff model we estimated the average density of the fluid in the turbulent cloud is in the range $1.8<\rho_{P} / \rho_{\text {air }}<4.0$. A summary of our investigation is shown in Figure 2. We have also shown that aerosols and droplets can remain suspended for hours in the air. Therefore, once the puff slows down sufficiently, and its coherence is lost, the eventual spreading of the infected aerosols becomes dependent on the ambient air currents and turbulence. De facto, as it was first pointed out in [19] and later developed in [29,30] airflow conditions strongly influence the distribution of viral particles in indoor spaces, cultivating a health threat from COVID-19 airborne infection.

Altogether, it seems reasonable to adopt additional infection-control measures for airborne transmission in highrisk settings, such as the use of face masks when in public. If the results of this study $-t_{f}$ of $O(h r)$ for aerosols, for example - are borne out by experiment, then these findings should be taken into account in policy decisions going forward as we continue to grapple with this pandemic.

\section{Funding}

The research of L.A.A. is supported by the U.S. National Science Foundation (NSF Grant PHY-1620661). J.B.D. acknowledges support from the National Science Foundation under Grant No. NSF PHY182080. The work of T.J.W. was supported in part by the U.S. Department of Energy (DoE grant No. DE- SC0011981).

\section{Role of the Funder/Sponsor}

The sponsors had no role in the preparation, review or approval of the manuscript and decision to submit the manuscript for publication. Any opinions, findings, and conclusions or recommendations expressed in this article are those of the authors and do not necessarily reflect the views of the NSF or DOE. 


\section{Declaration of Competing Interest}

The authors declare that they have no known competing financial interests or personal relationships that could have appeared to influence the work reported in this paper.

\section{Ethical Approval}

The manuscript does not contain experiments on animals and humans; hence ethical permission not required.

\section{References}

[1] Huang, Chaolin, Yeming Wang, Xingwang Li, Lili Ren, Jianping Zhao, Yi Hu, Li Zhang, et al. "Clinical Features of Patients Infected with 2019 Novel Coronavirus in Wuhan, China." The Lancet 395, no. 10223 (February 2020): 497-506. doi:10.1016/s0140-6736(20)30183-5.

[2] Zhou, Peng, Xing-Lou Yang, Xian-Guang Wang, Ben Hu, Lei Zhang, Wei Zhang, Hao-Rui Si, et al. "A Pneumonia Outbreak Associated with a New Coronavirus of Probable Bat Origin.” Nature 579, no. 7798 (February 3, 2020): $270-273$. doi:10.1038/s41586-020-2012-7.

[3] Zhu, Na, Dingyu Zhang, Wenling Wang, Xingwang Li, Bo Yang, Jingdong Song, Xiang Zhao, et al. "A Novel Coronavirus from Patients with Pneumonia in China, 2019.” New England Journal of Medicine 382, no. 8 (February 20, 2020): 727-733. doi:10.1056/nejmoa2001017.

[4] Tang, Xiaolu, Changcheng Wu, Xiang Li, Yuhe Song, Xinmin Yao, Xinkai Wu, Yuange Duan, et al. "On the Origin and Continuing Evolution of SARS-CoV-2." National Science Review 7, no. 6 (March 3, 2020): 1012-1023. doi:10.1093/nsr/nwaa036.

[5] Rothe, Camilla, Mirjam Schunk, Peter Sothmann, Gisela Bretzel, Guenter Froeschl, Claudia Wallrauch, Thorbjörn Zimmer, et al. "Transmission of 2019-nCoV Infection from an Asymptomatic Contact in Germany." New England Journal of Medicine 382, no. 10 (March 5, 2020): 970-971. doi:10.1056/nejmc2001468.

[6] Shiu, Eunice Y.C., Nancy H.L. Leung, and Benjamin J. Cowling. "Controversy Around Airborne Versus Droplet Transmission of Respiratory Viruses." Current Opinion in Infectious Diseases 32, no. 4 (August 2019): 372-379. doi:10.1097/qco.0000000000000563.

[7] Ong, Sean Wei Xiang, Yian Kim Tan, Po Ying Chia, Tau Hong Lee, Oon Tek Ng, Michelle Su Yen Wong, and Kalisvar Marimuthu. "Air, Surface Environmental, and Personal Protective Equipment Contamination by Severe Acute Respiratory Syndrome Coronavirus 2 (SARS-CoV-2) From a Symptomatic Patient." JAMA 323, no. 16 (April 28, 2020 ): 1610. doi:10.1001/jama.2020.3227.

[8] Santarpia, Joshua L, Danielle N Rivera, Vicki Herrera, M. Jane Morwitzer, Hannah Creager, George W. Santarpia, Kevin K Crown, et al. "Aerosol and Surface Transmission Potential of SARS-CoV-2" (March 26, 2020). doi:10.1101/2020.03.23.20039446.

[9] Liu, Yuan, Zhi Ning, Yu Chen, Ming Guo, Yingle Liu, Nirmal Kumar Gali, Li Sun, et al. “Aerodynamic Analysis of SARSCoV-2 in Two Wuhan Hospitals.” Nature 582, no. 7813 (April 27, 2020): 557-560. doi:10.1038/s41586-020-2271-3.

[10] Cai, Jing, Wenjie Sun, Jianping Huang, Michelle Gamber, Jing Wu, and Guiqing He. "Indirect Virus Transmission in Cluster of COVID-19 Cases, Wenzhou, China, 2020." Emerging Infectious Diseases 26, no. 6 (June 2020): 1343-1345. doi:10.3201/eid2606.200412.

[11] Guo, Zhen-Dong, Zhong-Yi Wang, Shou-Feng Zhang, Xiao Li, Lin Li, Chao Li, Yan Cui, et al. "Aerosol and Surface Distribution of Severe Acute Respiratory Syndrome Coronavirus 2 in Hospital Wards, Wuhan, China, 2020." Emerging Infectious Diseases 26, no. 7 (July 2020): 1583-1591. doi:10.3201/eid2607.200885.

[12] Van Doremalen, Neeltje, Trenton Bushmaker, Dylan H. Morris, Myndi G. Holbrook, Amandine Gamble, Brandi N. Williamson, Azaibi Tamin, et al. "Aerosol and Surface Stability of SARS-CoV-2 as Compared with SARS-CoV-1." New England Journal of Medicine 382, no. 16 (April 16, 2020): 1564-1567. doi:10.1056/nejmc2004973.

[13] Wölfel, Roman, Victor M. Corman, Wolfgang Guggemos, Michael Seilmaier, Sabine Zange, Marcel A. Müller, Daniela Niemeyer, et al. "Virological Assessment of Hospitalized Patients with COVID-2019." Nature 581, no. 7809 (April 1, 2020): 465-469. doi:10.1038/s41586-020-2196-x.

[14] Jones, Terry C, Barbara Mühlemann, Talitha Veith, Guido Biele, Marta Zuchowski, Jörg Hoffmann, Angela Stein, Anke Edelmann, Victor Max Corman, and Christian Drosten. "An Analysis of SARS-CoV-2 Viral Load by Patient Age" (June 9, 2020). doi:10.1101/2020.06.08.20125484.

[15] Bourouiba, Lydia. “A Sneeze.” New England Journal of Medicine 375, no. 8 (August 25, 2016): e15. doi:10.1056/nejmicm1501197. 
[16] Bourouiba, Lydia, Eline Dehandschoewercker, and John W. M. Bush. "Violent Expiratory Events: On Coughing and Sneezing.” Journal of Fluid Mechanics 745 (March 24, 2014): 537-563. doi:10.1017/jfm.2014.88.

[17] Scharfman, B. E., A. H. Techet, J. W. M. Bush, and L. Bourouiba. "Visualization of Sneeze Ejecta: Steps of Fluid Fragmentation Leading to Respiratory Droplets.” Experiments in Fluids 57, no. 2 (January 20, 2016). doi:10.1007/s00348015-2078-4.

[18] Bourouiba, Lydia. "Turbulent Gas Clouds and Respiratory Pathogen Emissions.” JAMA (March 26, 2020). doi:10.1001/jama.2020.4756.

[19] Anchordoqui, Luis Alfredo, and Eugene M. Chudnovsky. "A Physicist View of COVID-19 Airborne Infection through Convective Airflow in Indoor Spaces.” SciMedicine Journal 2 (August 28, 2020): 68-72. doi:10.28991/scimedj-2020-02-si-5.

[20] Gralton, Jan, Euan Tovey, Mary-Louise McLaws, and William D. Rawlinson. "The Role of Particle Size in Aerosolised Pathogen Transmission: A Review.” Journal of Infection 62, no. 1 (January 2011): 1-13. doi:10.1016/j.jinf.2010.11.010.

[21] Xie, X., Y. Li, A. T. Y. Chwang, P. L. Ho, and W. H. Seto. "How Far Droplets Can Move in Indoor Environments ? Revisiting the Wells Evaporation?falling Curve.” Indoor Air 17, no. 3 (June 2007): 211-225. doi:10.1111/j.1600-0668.2007.00469.x.

[22] Wei, Jianjian, and Yuguo Li. "Airborne Spread of Infectious Agents in the Indoor Environment." American Journal of Infection Control 44, no. 9 (September 2016): S102-S108. doi:10.1016/j.ajic.2016.06.003.

[23] Cole, Eugene C., and Carl E. Cook. "Characterization of Infectious Aerosols in Health Care Facilities: An Aid to Effective Engineering Controls and Preventive Strategies.” American Journal of Infection Control 26, no. 4 (August 1998): $453-464$. doi:10.1016/s0196-6553(98)70046-x.

[24] Duguid, J. P. "The Size and the Duration of Air-Carriage of Respiratory Droplets and Droplet-Nuclei." Epidemiology and Infection 44, no. 6 (September 1946): 471-479. doi:10.1017/s0022172400019288.

[25] Yang, Shinhao, Grace W.M. Lee, Cheng-Min Chen, Chih-Cheng Wu, and Kuo-Pin Yu. "The Size and Concentration of Droplets Generated by Coughing in Human Subjects." Journal of Aerosol Medicine 20, no. 4 (December 2007): $484-494$. doi:10.1089/jam.2007.0610.

[26] Balachandar, S., S. Zaleski, A. Soldati, G. Ahmadi, and L. Bourouiba. "Host-to-Host Airborne Transmission As a Multiphase Flow Problem For Science-Based Social Distance Guidelines.” International Journal of Multiphase Flow (September 2020): 103439. doi:10.1016/j.ijmultiphaseflow.2020.103439.

[27] Morton, B. R. Taylor, G. I. \& Turner, J. S. “Turbulent Gravitational Convection from Maintained and Instantaneous Sources.” Proceedings of the Royal Society of London. Series A. Mathematical and Physical Sciences 234, no. 1196 (January 24, 1956): 1-23. doi:10.1098/rspa.1956.0011.

[28] Wells, William F. "On air-borne infection. Study II. Droplets and droplet nuclei." American Journal of Hygiene 20 (1934): 611-18.

[29] Augenbraun, Benjamin L., Zack D. Lasner, Debayan Mitra, Sridhar Prabhu, Shivam Raval, Hiromitsu Sawaoka, and John M. Doyle. "Assessment and Mitigation of Aerosol Airborne SARS-CoV-2 Transmission in Laboratory and Office Environments." Journal of Occupational and Environmental Hygiene 17, no. 10 (September 22, 2020): 447-456. doi:10.1080/15459624.2020.1805117.

[30] Evans, Matthew. “Avoiding COVID-19: Aerosol Guidelines” (May 25, 2020). doi:10.1101/2020.05.21.20108894.

[31] Crowder, Timothy M., Jacky A. Rosati, Jeffry D. Schroeter, Anthony J. Hickey, and Ted B. Martonen. "Fundamental effects of particle morphology on lung delivery: predictions of Stokes' law and the particular relevance to dry powder inhaler formulation and development." Pharmaceutical research 19, no. 3 (2002): 239-245. doi:10.1023/A:1014426530935. 


\section{Appendix I}

There are important considerations in the development of Stokes' law, including the hypothesis that the gas at particle surface has zero velocity relative to the particle. This hypothesis holds well when the diameter of the particle is much larger than the mean free path of gas molecules. The mean free path $\lambda_{m f p}^{\text {air }}$ is the average distance travelled by a gas molecule between two successive collisions. In analyses of the interaction between gas molecules and particles, it is convenient to use the Knudsen number $K_{n}=2 \lambda_{m f p}^{\text {air }} / D_{V}$ a dimensionless number defined as the ratio of the mean free path to particle radius. For $\mathrm{Kn} \gtrsim 1$, the drag force is smaller than predicted by Stokes' law. Conventionally this condition is described as a result of slip on the particle surface. The so-called slip correction is estimated to be [31]:

$\kappa=1+\mathrm{Kn}[1.257+0.4 \exp (-1.1 / \mathrm{Kn}]$

In our calculations we take:

$\lambda_{\text {mfp }}^{\text {air }}=\frac{\eta_{\text {air }}}{\rho_{\text {air }}}\left(\frac{\pi m_{\text {air }}}{2 k_{\mathrm{B}} T}\right)^{1 / 2}$

Where $k_{B}$ is the Boltzmann constant, $T$ is the temperature in Kelvin, and the density of air is given by:

$\rho_{\text {air }}=\frac{P}{R_{g} T}$

With $P=101 \mathrm{kPa}$, and where $R_{g}=287.058 \mathrm{~J} /(\mathrm{kg} . \mathrm{K})$ is the ideal gas constant. The molar mass of air is $m_{m o l}=$ $29 \mathrm{~g} / \mathrm{mol}$, which leads to $m_{\text {air }}=4.8 \times 10^{-26} \mathrm{~kg} / \mathrm{molecule}$. 